\title{
On Use of the Standard Deviation of the Mass Distribution as a Parameter in Raindrop Size Distribution Functions
}

\author{
PAUL L. SMITH \\ Atmospheric and Environmental Sciences Program, South Dakota School of Mines and Technology, \\ Rapid City, South Dakota \\ ROGER W. JOHNSON AND DONNA V. KLICHE \\ Department of Mathematics and Computer Science, South Dakota School of Mines and Technology, \\ Rapid City, South Dakota
}

(Manuscript received 28 March 2018, in final form 24 September 2018)

\begin{abstract}
Use of the standard deviation $\sigma_{m}$ of the drop mass distribution as one of the three parameters of raindrop size distribution (DSD) functions was introduced for application to disdrometer data supporting the Global Precipitation Measurement dual-frequency radar system. The other two parameters are a normalized drop number concentration $N_{w}$ and the mass-weighted mean diameter $D_{m}$. This paper presents an evaluation of that formulation of the DSD functions, in two parts. First is a mathematical analysis showing that the procedure for estimating $\sigma_{m}$, along with the other DSD parameters, from disdrometer data is in essence another moment method. As such, it is subject to the biases and errors inherent in all moment methods. When the form of the DSD function is specified, it is inferior (like all moment methods) to the maximum likelihood technique for fitting parameters to sampled data. The second part is a series of sampling simulations illustrating the biases and errors involved in applying the formulation to the specific case of gamma DSDs. It leads to underestimates of $\sigma_{m}$ and consequently to overestimates of the gamma shape parameter-with large root-mean-square errors. Comparison with maximum likelihood estimates shows the degree of improvement that could be obtained in the estimates of the shape parameter. The propensity to underestimate $\sigma_{m}$ will be pervasive, and users of this DSD formulation should be cognizant of the biases and errors that can occur.
\end{abstract}

\section{Introduction}

Mathematical models of raindrop size distribution (DSD) functions are useful in a variety of fields, including radar and satellite meteorology, cloud and precipitation physics, and numerical cloud modeling. The earliest model of the DSDs in the atmosphere was the exponential form introduced by Marshall and Palmer (1948). Later models have included the widely used gamma distribution (e.g., Ulbrich 1983; Bringi and Chandrasekar 2001), the lognormal distribution (e.g., Bradley and Stow 1974; Markowitz 1976; Feingold and Levin 1986), and the Weibull distribution (e.g., Best 1950; Wilks 1989; Alonge and Afullo 2012). Recently Williams et al. (2014) introduced a model that uses the standard deviation $\sigma_{m}$ of the drop mass distribution as

Corresponding author: Paul L. Smith, paul.smith@sdsmt.edu a parameter, the other two parameters being a normalized drop number concentration $N_{w}$ and the massweighted mean diameter $D_{m}$. This model was devised for use with disdrometer data supporting the Global Precipitation Measurement (GPM) mission dual-frequency precipitation radar system (Hou et al. 2014); it has also been used elsewhere (e.g., Thurai et al. 2017). The use of $\sigma_{m}$ as a DSD parameter is unusual [although it is mentioned in Ulbrich and Atlas (1998) and Bringi and Chandrasekar (2001)], but use of a measure of the width of the distribution as a parameter is not without precedent. One of the parameters in the lognormal distribution sometimes used for raindrop distribution functions (e.g., Feingold and Levin 1986) is the width of the distribution.

Regardless of the model used, a means of estimating the parameters for the model from observations of drop size distributions is typically needed. This is in essence a 
statistical problem of fitting a distribution function to a data sample collected from a population of drops-the population being the drops contained in some volume of the atmosphere that is of interest. That volume typically has dimensions of at least hundreds of meters and contains upward of $10^{8}$ drops, whereas available disdrometer instrumentation typically samples no more than a few thousand drops (and often far fewer). The preferred statistical method of fitting distributions to data is the maximum likelihood method (e.g., Rice 1995; Mallet and Barthes 2009); the moment methods that have been widely used in the atmospheric science literature (e.g., Waldvogel 1974; Weber 1976; Ulbrich 1983; Kozu and Nakamura 1991; Tokay and Short 1996; Ulbrich and Atlas 1998; Caracciolo et al. 2006) are not efficient and are decidedly inferior in terms of bias and variability of the parameter estimates (e.g., Robertson and Fryer 1970; Wallis et al. 1974; Smith et al. 2009; Ye and Chen 2017).

The present work concerns the use of the standard deviation of the drop mass distribution as a DSD parameter, the associated method of fitting the parameters to disdrometer data, and their application to the specific case of gamma DSD functions. Section 2 outlines the basic DSD formulation and the associated parameter-fitting method, and shows that the latter is essentially a moment method subject to the biases and errors involved in any moment method. Section 3 assesses the method for the case of gamma DSDs via a sampling simulation procedure and compares it with the optimum maximum likelihood estimators. Discussion and conclusions appear in section 4 .

\section{The DSD formulation and parameter estimation procedure}

The primary reason for focusing on the distribution of raindrop mass, rather than number, with size is to circumvent difficulties with observing small drops (Williams et al. 2014). The small drops may be quite numerous but contribute little to the overall population mass (or to other quantities of practical interest, such as rainfall rate or radar reflectivity). The Williams et al. approach does not require assuming a specific form for the DSD functions (see also Williams et al. 2015), and for purposes of the present discussion a "generic" form of the DSD functions will be useful as a point of departure. Their work makes use of the gamma distribution for specific examples, and we shall do the same in section 3.

The generic form of the DSD functions can, after introduction of $\sigma_{m}$ as a parameter, be written as

$$
n(D)=N_{W} f_{1}\left(D ; D_{m}, \sigma_{m}\right) .
$$

Here $n(D)$ represents the number of drops per unit volume per unit size interval and $D$ is the equivalent spherical drop diameter. This form defines a family of DSD functions, rather than an individual one; to show this, note that the generic form of the functions can also be written as

$$
n(D)=N_{T} f_{2}\left(D ; D_{m}, \sigma_{m}\right) .
$$

Here $N_{T}$ is the drop number concentration and the function $f_{2}$ is the probability density function (PDF) of drop diameter. The function $f_{1}$ is not a PDF, because it is dimensionless and its integral over all $D$ is not unity; Williams et al. (2014) refer to it as a "quasi PDF."

To introduce the parameter $N_{W}$ into Eq. (2), we follow the lead of Sekhon and Srivastava (1970) in first making the equation dimensionless by multiplying both sides by $\rho D_{m}^{4} / W$; here $\rho$ is the density of water and $W$ is the liquid water concentration. The value of $W$ is

$$
W=\pi \rho N_{T} D_{m}^{3} /(6 a),
$$

with the value of the numerical factor $a$ depending upon the specific form and parameters of the DSD function. Rearranging factors, the expression for $n(D)$ becomes identical to Eq. (1) with the normalized coefficient $N_{w}$ being

$$
N_{w}=\frac{256 W}{\pi \rho D_{m}^{4}}\left(=\frac{128 N_{T}}{3 a D_{m}}\right) .
$$

This form shows that numerous DSD functions can have the same values of the three parameters $N_{w}, D_{m}$, and $\sigma_{m}$, as long as the ratio $N_{T} / a$ is the same.

The parameters of this DSD function can be estimated from disdrometer data. An estimator for $D_{m}$ follows from the definition as

$$
\hat{D}_{m}=\frac{\sum N(D) D^{4} d D}{\sum N(D) D^{3} d D} .
$$

Here $N(D) d D$ is the observed concentration of drops in the size interval $D \rightarrow D+d D$, and the summations are carried out over all of the observed drop sizes. A straightforward estimator for $N_{w}$ follows from Eqs. (4) and (5) as

$$
\hat{N}_{w}=\frac{128\left[\sum N(D) D^{3} d D\right]^{5}}{3\left[\sum N(D) D^{4} d D\right]^{4}} .
$$


In the Williams et al. (2014) work values of $N_{w}$ are estimated from disdrometer data after first determining empirical relationships among the DSD parameters; the $N_{w}$ estimator [their Eq. (27)] also involves the estimate of the equivalent radar reflectivity factor $Z_{e}$, which entails another summation over the observed drop spectrum.

The expressions in Eqs. (5) and (6) are moment estimators, as is the Williams et al. (2014) estimator for $N_{w}$. The estimator for $\sigma_{m}$ follows Williams et al.'s Eq. (15):

$$
\hat{\sigma}_{m}^{2}=\frac{\sum\left(D-\hat{D}_{m}\right)^{2} N(D) D^{3} d D}{\sum N(D) D^{3} d D} .
$$

To show that this is also a moment estimator requires some algebra. After expanding the numerator and separating the terms,

$$
\begin{aligned}
\hat{\sigma}_{m}^{2} & =\frac{\sum N(D) D^{5} d D}{\sum N(D) D^{3} d D}-2 \hat{D}_{m} \frac{\sum N(D) D^{4} d D}{\sum N(D) D^{3} d D}+\hat{D}_{m}^{2} \frac{\sum N(D) D^{3} d D}{\sum N(D) D^{3} d D} \\
& =\frac{\sum N(D) D^{5} d D}{\sum N(D) D^{3} d D}-\hat{D}_{m}^{2} .
\end{aligned}
$$

Let the $i$ th moment of the sample distribution be denoted by $m_{i}$; then Eq. (8) reduces to

$$
\hat{\sigma}_{m}^{2}=\frac{m_{3} m_{5}-m_{4}^{2}}{m_{3}^{2}} .
$$

This is clearly another moment estimator and as such will be subject to the biases and errors inherent in all moment-method estimators (e.g., Robertson and Fryer 1970; Wallis et al. 1974; Smith et al. 2009). Those biases and errors tend to increase with the order of the moments employed, and therefore on that score it is not a particularly good moment estimator. The veracity of that statement can be illustrated by the specific case of gamma distributions, as discussed in the next section.

\section{Example of gamma distributions}

\section{a. Analysis and summary of earlier results}

One form of the gamma DSD function is

$$
n_{g}(D)=N_{T} \frac{(\mu+4)^{\mu+1}}{\Gamma(\mu+1)} \frac{D^{\mu}}{D_{m}^{\mu+1}} \exp \left[-\frac{(\mu+4) D}{D_{m}}\right] .
$$

Here $\mu$ is the dimensionless gamma shape parameter. In the absence of truncation of the distribution at either end, the $i$ th moment of this distribution is

$$
M_{i g}=N_{T} \frac{(\mu+1)(\mu+2) \cdots(\mu+i)}{(\mu+4)^{i}} D_{m}^{i},
$$

and the factor $a$ in Eq. (3) is

$$
a_{g}=\frac{(\mu+4)^{3}}{(\mu+1)(\mu+2)(\mu+3)} .
$$

Williams et al. (2014) note that the standard deviation $\sigma_{m g}$ of the mass spectrum for an untruncated gamma distribution is related to the shape parameter by

$$
\sigma_{m g}^{2}=\frac{D_{m}^{2}}{(\mu+4)} \quad \text { or } \quad \mu=\frac{D_{m}^{2}}{\sigma_{m g}^{2}}-4 .
$$

The estimators in Eqs. (5), (6), and (9) use sample moments $m_{3}, m_{4}$, and $m_{5}$, and the Williams et al. (2014) estimator for $N_{w}$ adds $m_{6}$ to the mix. The "conventional" moment estimators for the gamma DSD parameters using sample moments $m_{3}, m_{4}$, and $m_{5}$ would be identical to Eqs. (5) and (6) for $\hat{D}_{m}$ and $\hat{N}_{w}$, respectively, and

$$
\hat{\mu}=\frac{5 m_{4}^{2}-4 m_{3} m_{5}}{m_{3} m_{5}-m_{4}^{2}}
$$

for the shape parameter. The latter is identical to the result obtained by combining Eqs. (5), (9), and (13). So in essence the estimators presented in Williams et al., for the gamma case at least, are just moment estimators using sample moments $m_{3}, m_{4}$, and $m_{5}$ (and $m_{6}$ ) presented in a different form.

The biases and errors in moment estimators for the parameters of gamma DSDs have been evaluated in Kliche (2007) and Smith et al. (2009)—although the specific case of the combination $m_{3}, m_{4}$, and $m_{5}$ was not included and the parameter $\sigma_{m g}$ was not considered. The treatment was extended to left-truncated data samples in Johnson et al. (2011) and to data samples truncated on both ends as well as binned in Johnson et al. (2014). The general tendency is for the moment methods to underestimate $D_{m}$ and $N_{T}$ while overestimating $N_{w}$ and $\mu$; the relative biases and errors 
increase in the order in which those parameters are listed. Especially for small samples, the parameter estimates are very sensitive to the size of the largest drop in the sample. The biases and errors increase with the order of the moments employed, are smaller for narrower gamma distributions, and decrease as the data sample size increases. Regardless of the specific moment combination employed or the specific gamma DSD parameters, the moment method is inferior to the maximum likelihood procedure (e.g., Rice 1995).

\section{b. Simulations relative to the use of $\sigma_{m}$ as a gamma DSD parameter}

To illustrate the biases and errors involved in estimating from disdrometer data the parameters for the gamma DSD formulation using $\sigma_{m}$ as a parameter, we carried out sampling simulations analogous to those described in Smith et al. (2009). We postulated a variety of gammadistributed raindrop populations; to simplify the simulations we then generated random volume samples. Johnson et al. (2014) derived a relation between volume and surface samples, and an example appears in the appendix of Smith (2016). In brief, a gamma DSD in the atmosphere yields another gamma DSD on the surface, with different parameters. As a result, the sampling statistics for volume samples from a gamma DSD with parameters $\mu$ and $D_{m}$ correspond to those for surface samples from a gamma DSD with parameters $\mu^{\prime}=\mu-1$ and

$$
D_{m}^{\prime}=\frac{(\mu+4) D_{m}}{\mu+5-\left(0.193 \mathrm{~mm}^{-1}\right) D_{m}} .
$$

For example, the results below for $\mu=2$ and any value of $D_{m}$ (say, $D_{m}=1.5 \mathrm{~mm}$ ) would apply for surface disdrometer samples from a gamma DSD with $\mu^{\prime}=1$ and a somewhat smaller value of $D_{m}\left(D_{m}^{\prime}=1.34 \mathrm{~mm}\right.$ in this example).

We organized the results in terms of sample sizes rather than drop number concentrations, and here we use fixed sample sizes instead of the Poisson-distributed sample sizes employed earlier. We sampled from distributions with $\mu=2,5$, and 10; as noted in Smith et al. (2009), the drop sizes in Eq. (10) can be normalized in the form $D / D_{m}$ so that

$$
\begin{aligned}
n_{g}\left(\frac{D}{D_{m}}\right)= & N_{T} \frac{(\mu+4)^{\mu+1}}{\Gamma(\mu+1)}\left(\frac{D}{D_{m}}\right)^{\mu} \\
& \times \exp \left[-(\mu+4)\left(\frac{D}{D_{m}}\right)\right] .
\end{aligned}
$$

Then, in the absence of truncation, the sampling statistics are independent of $D_{m}$ and simulation runs for different values of $D_{m}$ are not needed. (In some places we have used the value $D_{m}=1.5 \mathrm{~mm}$ for purposes of illustration.)

The postulated population DSDs are not truncated; there is no real basis for assuming that small drops are not present, and extremely large drops are so rare in the gamma DSDs that excluding them would have no significant effect on the simulation results. Truncation of the sample data was not included in the simulations; to do so would complicate the simulation procedure and, with the tendency being to underestimate $\sigma_{m g}$, any such truncation in actual data would exacerbate that tendency and work to increase the biases we find. Moreover, the data truncation introduces an asymptotic bias. Incorporating data truncation would cause the details of the results to vary but would not impact the general sense of conclusions about biases and errors or their dependence upon population DSD parameters or sample sizes.

Our estimates of the DSD parameters $N_{w}, \mu, D_{m}$, and $\sigma_{m}$ were based on sample moments $m_{3}, m_{4}$, and $m_{5}$ and used Eqs. (5), (6), (9), and (14). For comparison, we also include the corresponding maximum likelihood estimates of parameters $\mu$ and $D_{m}$. Williams et al. (2014) used data with a minimum sample size of 50 drops; Smith et al. (2009) included results for sample sizes of 50 and 1000 drops, and we do the same here. For each set of parameters and sample sizes we used 1000 iterations; plots of cumulative distributions show only every 20th value so that the symbols are clearer. Some additional results for mean sample sizes covering the range from 10 to 1000 drops appear in Kliche (2007).

\section{1) RESULTS FOR SAMPLES OF 50 DROPS FROM POPULATION $\mu=2$}

The sampling distributions of the moments of a DSD are unbiased but highly skewed, the skewness increasing with the order of the moment considered. The parameter $\sigma_{m}$ is not a simple DSD moment but is estimated from moments $m_{3}, m_{4}$, and $m_{5}$ as shown in Eq. (9); its sampling distribution is also strongly skewed, with more than $80 \%$ of the sample values being underestimates in this case. Figure 1 shows cumulative frequency distributions of the normalized estimates of these quantities to illustrate this skewness.

The mass distribution for samples as small as 50 drops is irregular. Figure 2 shows an example of such a distribution along with the corresponding population distribution; the figure caption includes the estimates of the DSD parameters as based on this sample. The rightmost bin $(2.4 \mathrm{~mm})$ contained a single drop, illustrating the importance of the largest drop in the sample [a topic that is discussed in more detail in section $3 b(3)]$. 


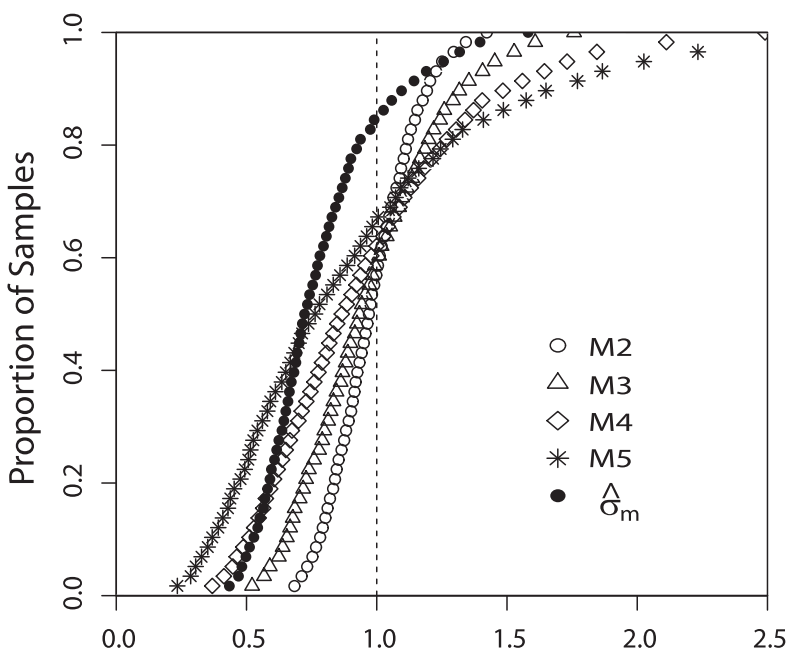

Ratio of Sample Value to Population Value

FIG. 1. Illustration of skewness in the sampling distributions of normalized sample moments $m_{i}(i=2,3,4,5)$ and normalized estimates of the mass spectrum standard deviation $\hat{\sigma}_{m}$. Population shape parameter $\mu=2$; sample sizes are 50 drops.

Figure 3 shows the sampling distributions for the normalized estimates of $D_{m}, \sigma_{m}, N_{w}$, and $\mu$ for samples of 50 drops from a gamma population with $\mu=2$. The tendencies to underestimate $D_{m}$ and overestimate $N_{w}$ and $\mu$ are evident; as noted above the procedure also tends to underestimate $\sigma_{m}$ (which would be consistent with the overestimates of $\mu$ ). The reason for the wide variability in the estimates of $\mu$ can be seen by rearranging Eq. (14); let

$$
\eta_{g}=\frac{m_{3 g} m_{5 g}}{m_{4 g}^{2}}=\frac{\mu+5}{\mu+4} .
$$

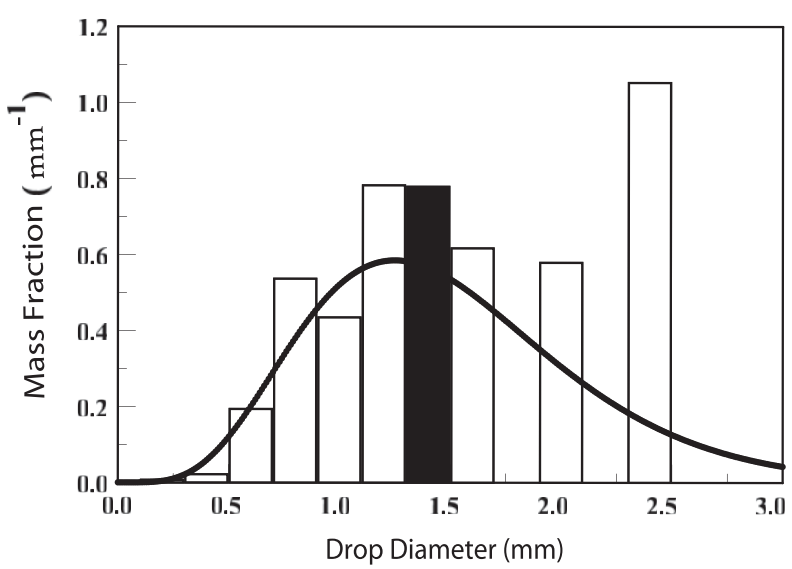

FIG. 2. Example of the mass distribution for a sample of only 50 drops from a gamma DSD with population parameters $\mu=2$ and $D_{m}=1.5 \mathrm{~mm}$; bin widths for drop sizes are $0.2 \mathrm{~mm}$. Sample estimates of the parameters are $\hat{\mu}=4.08$ and $\hat{D}_{m}=1.52 \mathrm{~mm}$; for the standard deviation, the estimate $\hat{\sigma}_{m}$ is $87 \%$ of the population value.

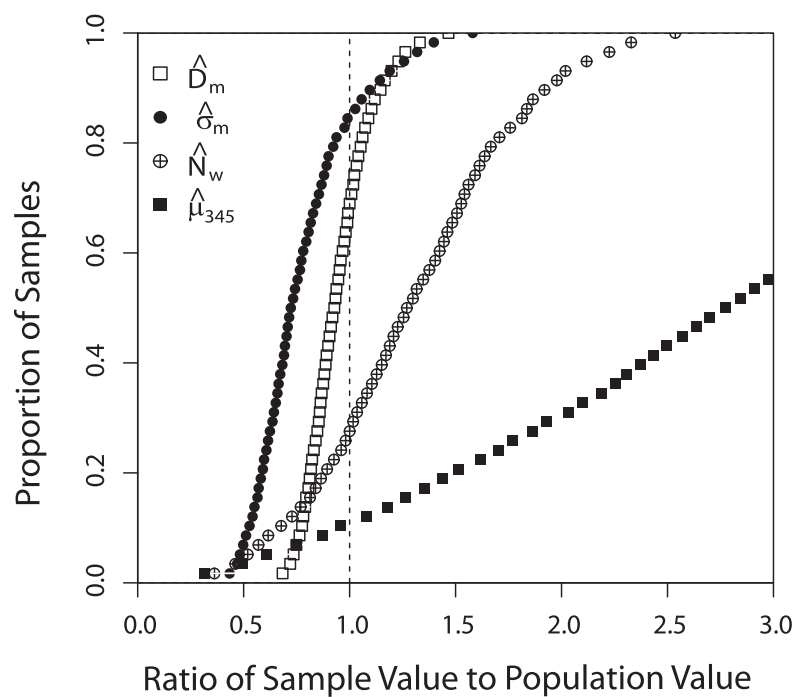

FIG. 3. Illustration of skewness in the distributions of sample estimates of gamma DSD parameters $D_{m}, \sigma_{m}, N_{w}$, and $\mu$ according to the scheme outlined in the text, and the tendency to underestimate $\sigma_{m}$ and overestimate $\mu$. Cumulative frequency distributions are of normalized sample estimates; population shape parameter $\mu=2$; sample sizes are 50 drops.

Then Eq. (14) becomes

$$
\hat{\mu}=\frac{1}{\hat{\eta}_{g}-1}-4
$$

For values of $\mu$ ranging from 0 to 10 [the range that was considered by Williams et al. (2014)], the value of $\eta_{g}$ varies from 1.25 to 1.07 . With $\hat{\eta}_{g}-1$ in the denominator of Eq. (18), small variations in the sample values of $\eta_{g}$ produce large variations in the estimates of $\mu$.

The sample estimates of $\sigma_{m}$ and $D_{m}$ are strongly correlated, as illustrated in Fig. 4; the correlation coefficient is 0.92 . This results from the fact that both estimates are functions of the sample moments $m_{3}$ and $m_{4}$.

As mentioned earlier, moment methods are inferior to maximum likelihood procedures for estimating DSD parameters when the form of the function is specified. Figure 5 clearly illustrates this for the gamma DSD with parameters $N_{w}, D_{m}$, and $\sigma_{m}$. The maximum likelihood estimates of $\mu$ have small bias (mean factor 1.10) with root-mean-square error (RMSE) 0.33. In contrast, the moment estimates are strongly biased (mean factor 3.01) with an RMSE of 2.58 .

\section{2) RESUlTS FOR SAMPLES OF 1000 DROPS FROM POPULATION $\mu=2$}

With larger sample sizes, the estimates of all of the parameters improve (e.g., Fig. 6). However, even with samples of 1000 drops almost $2 / 3$ of the $\hat{\sigma}_{m}$ values are still 


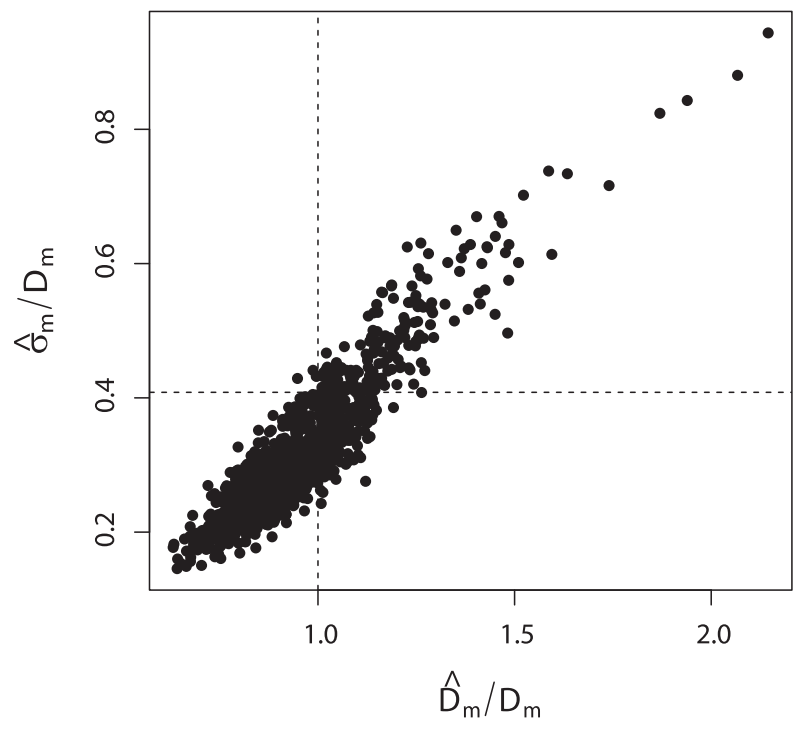

FIG. 4. Illustration of the statistical correlation between sample estimates of DSD parameters $\hat{\sigma}_{m}$ and $\hat{D}_{m}$; samples are of 50 drops from a gamma DSD with population shape parameter $\mu=2$. Dashed lines indicate the normalized population values.

underestimates and the moment estimates of $\mu$ remain strongly skewed and biased; the bias in $\hat{\mu}$ is more than $25 \%$ with an RMSE of $61 \%$. The bias in the corresponding maximum likelihood estimates is less than $1 \%$ with an RMSE of only 13\%; Fig. 7 illustrates the

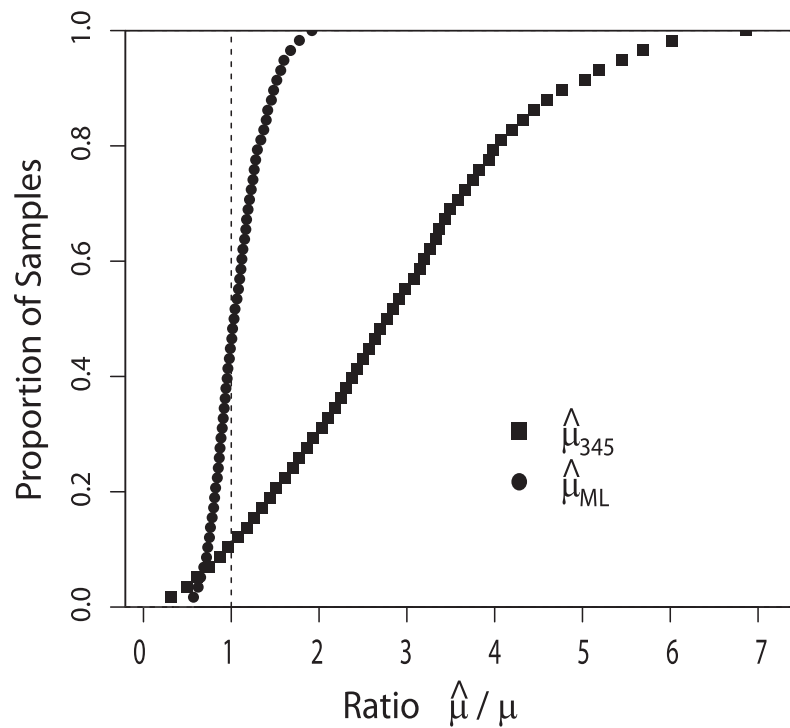

FIG. 5. Illustration of superiority of the maximum likelihood method; cumulative frequency distributions are of normalized sample estimates of gamma DSD shape parameter $\mu$ from Eq. (13) $\left(\hat{\mu}_{345}\right)$ and the maximum likelihood method $\left(\hat{\mu}_{\mathrm{ML}}\right)$. Population shape parameter $\mu=2$; sample sizes are 50 drops.

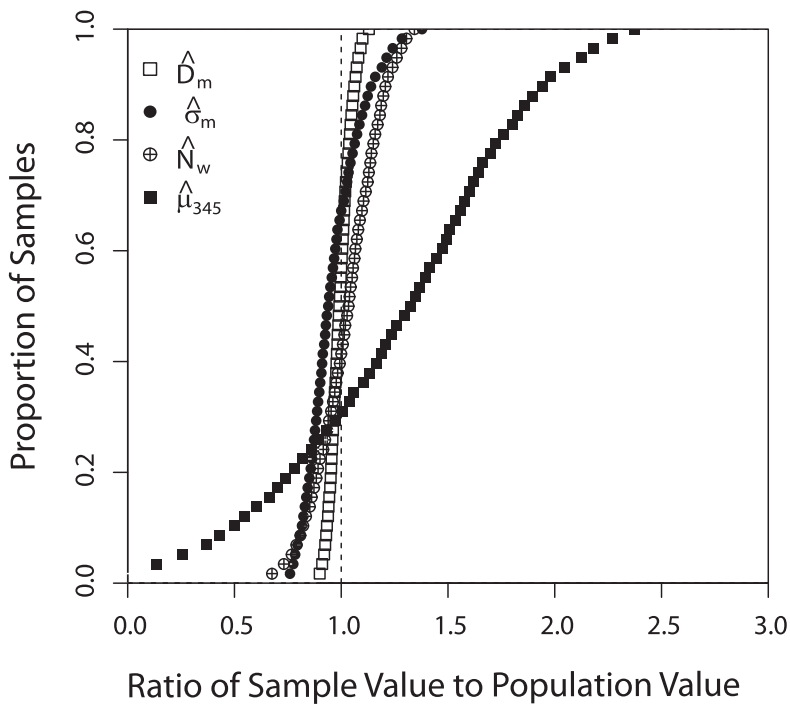

FIG. 6. Illustration of skewness in the distributions of sample estimates of gamma DSD parameters $D_{m}, \sigma_{m}, N_{w}$, and $\mu$ according to the scheme outlined in the text, with large sample sizes. Cumulative frequency distributions are of normalized sample estimates; population shape parameter $\mu=2$; sample sizes are 1000 drops.

superiority of the maximum likelihood estimates even with this large sample size.

Table 1 illustrates the overall effects of sample size on the gamma parameter estimates. With sample sizes of 1000 drops the biases in the moment estimators are, with

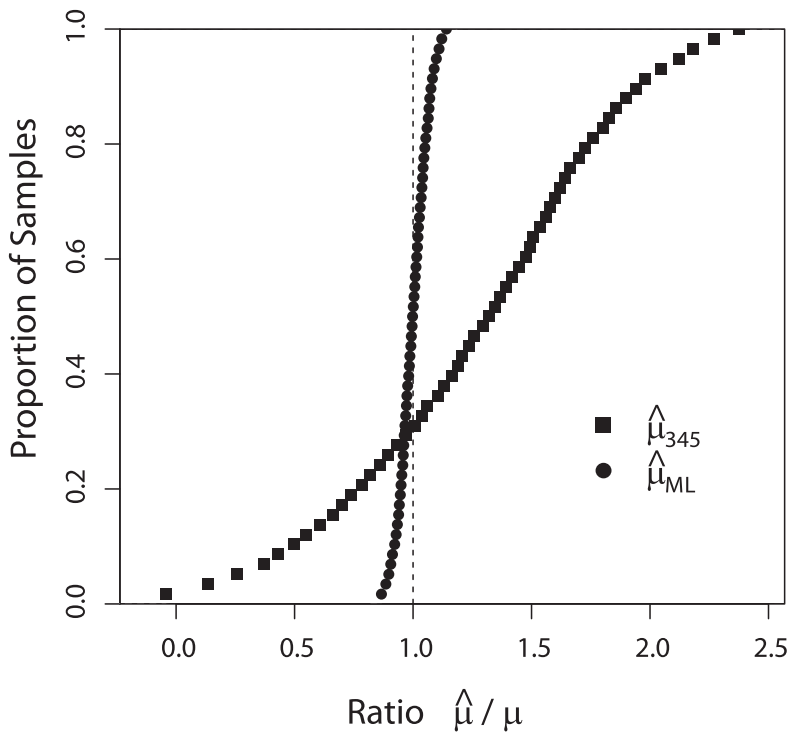

FIG. 7. Illustration of superiority of the maximum likelihood method with large sample sizes; cumulative frequency distributions are of normalized sample estimates of gamma DSD parameter $\mu$ from Eq. (13) $\left(\hat{\mu}_{345}\right)$ and the maximum likelihood method $\left(\hat{\mu}_{\mathrm{ML}}\right)$. Population shape parameter $\mu=2$; sample sizes are 1000 drops. 
TABLE 1. Summary statistics for the normalized Eq. (13) (m345) and maximum likelihood estimates of the indicated gamma DSD parameters. Population parameter $\mu=2$.

\begin{tabular}{|c|c|c|c|c|c|c|c|}
\hline \multirow[b]{2}{*}{ Sample size } & \multirow[b]{2}{*}{ Estimate } & \multicolumn{3}{|c|}{ m345 estimators } & \multicolumn{3}{|c|}{ Max likelihood estimators } \\
\hline & & Median & Mean & RMSE & Median & Mean & RMSE \\
\hline \multirow[t]{4}{*}{50} & $\hat{\mu} / \mu$ & 2.78 & 3.01 & 2.58 & 1.06 & 1.10 & 0.33 \\
\hline & $\hat{\sigma}_{m} / \sigma_{m}$ & 0.731 & 0.781 & 0.329 & - & - & - \\
\hline & $\hat{N}_{w} / N_{w}$ & 1.30 & 1.33 & 0.610 & - & - & - \\
\hline & $\hat{D}_{m} / D_{m}$ & 0.926 & 0.953 & 0.169 & 0.980 & 0.987 & 0.120 \\
\hline \multirow[t]{4}{*}{1000} & $\hat{\mu} / \mu$ & 1.29 & 1.25 & 0.613 & 1.003 & 1.006 & 0.065 \\
\hline & $\hat{\sigma}_{m} / \sigma_{m}$ & 0.947 & 0.973 & 0.142 & - & - & - \\
\hline & $\hat{N}_{w} / N_{w}$ & 1.027 & 1.026 & 0.148 & - & - & - \\
\hline & $\hat{D}_{m} / D_{m}$ & 0.993 & 0.997 & 0.050 & 0.999 & 0.999 & 0.028 \\
\hline
\end{tabular}

the exception of that in the shape parameter $\mu$, reduced to less than $3 \%$. However, the RMSEs are still substantial and the advantages of the maximum likelihood estimates are apparent (there are no maximum likelihood estimators for $N w$ or $\sigma_{m}$ ). Comparison with Table 1 of Smith et al. (2009) shows that the biases and errors of the estimators for the formulation with parameters $N_{w}, D_{m}$, and $\sigma_{m}$ (the "M345" estimates) are intermediate between those of the "M234" and "M346" estimators considered in that paper. The correlation between the estimates of $\hat{\sigma}_{m}$ and $\hat{D}_{m}$ remains strong (coefficient $r=0.88$ ), and the values for a sample size of 1000 reasonably overlap those for a sample size of 50 (e.g., Fig. 8).

\section{3) ISSUES WITH SAMPLING THE LARGE-DROP TAIL OF THE DSD}

Samples as small as 50 drops suffer from great difficulty in obtaining an adequate sample of the large drops in the population, and the difficulties carry over for larger sample sizes as well. The values of the sample moments and of quantities estimated therefrom consequently exhibit strong dependence on the size of the largest drop in the sample $D_{\max }$. Figure 9 illustrates this behavior for the sample estimates of the mass standard deviation $\hat{\sigma}_{m}$; the correlation coefficient for a sample size of 50 drops is 0.96 , and for 1000 drops it remains at 0.92 .

This in turn leads to estimates of the gamma shape parameter $\mu$ that are correlated with $D_{\max }$ (e.g., Fig. 10). The linear correlation coefficient here is -0.76 , although a nonlinear fit would have even stronger correlation. The correlation of the other estimated DSD parameters $\hat{D}_{m}$ and $\hat{N}_{w}$ with $D_{\max }$ is 0.9 or higher. With a sample size of 50 drops, even the maximum likelihood estimates of $D_{m}$ have a correlation of 0.58 with $D_{\max }$.

In effect, what the introduction of $\sigma_{m}$ as a DSD parameter has done is trade problems with observing the small raindrops for other problems in adequately sampling the large drops. Whether this results in a net benefit to the accuracy of the DSD parameter estimates is unclear.

\section{4) RESULTS FOR SAMPLES FROM POPULATIONS WITH OTHER VALUES OF $\mu$}

In this section we focus on the m345 estimates of $\sigma_{m}$ and $\mu$; sampling statistics for the other DSD parameters $\left(D_{m}\right.$ and $\left.N_{w}\right)$ are summarized in Smith et al. (2009), although no values for $\mu=10$ are included there. Figure 11 shows the sampling distributions of $\hat{\sigma}_{m}$ for population $\mu$ values of 2,5 , and 10 and sample sizes of 50 drops. The skewness of the distribution decreases slowly with increasing population values of $\mu$; even with $\mu=10$ more than $70 \%$ of the sample values are still underestimates. The bias is also reduced, although for $\mu=10$ it is still almost $-8 \%$, and the RMSE remains above $22 \%$.

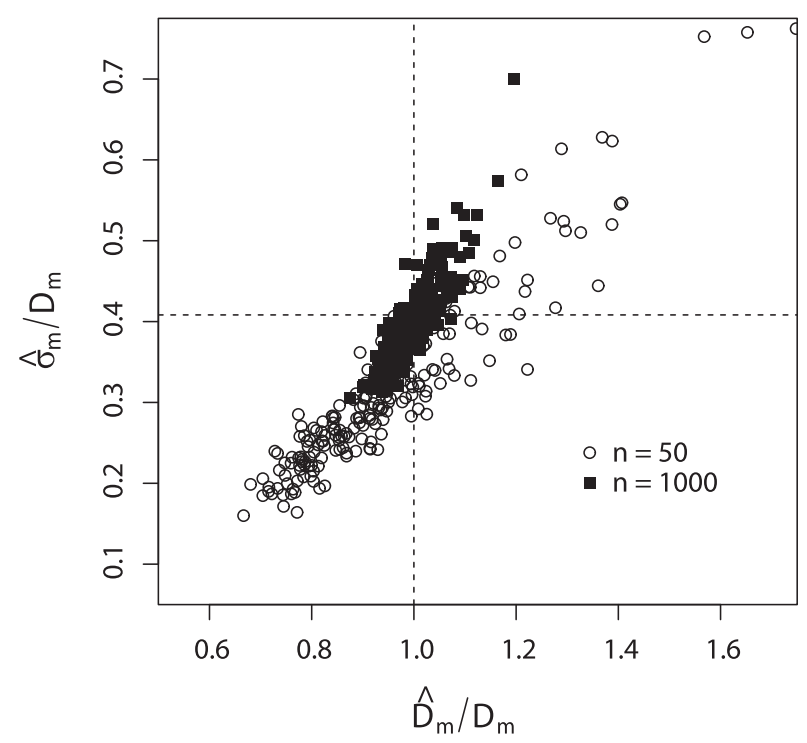

FIG. 8. Illustration of the statistical correlation between sample estimates of DSD parameter $\hat{\sigma}_{m}$ and $\hat{D}_{m}$ with varied sample sizes; samples are of 50 and 1000 drops from a gamma DSD with population shape parameter $\mu=2$. Dashed lines indicate the normalized population values. 


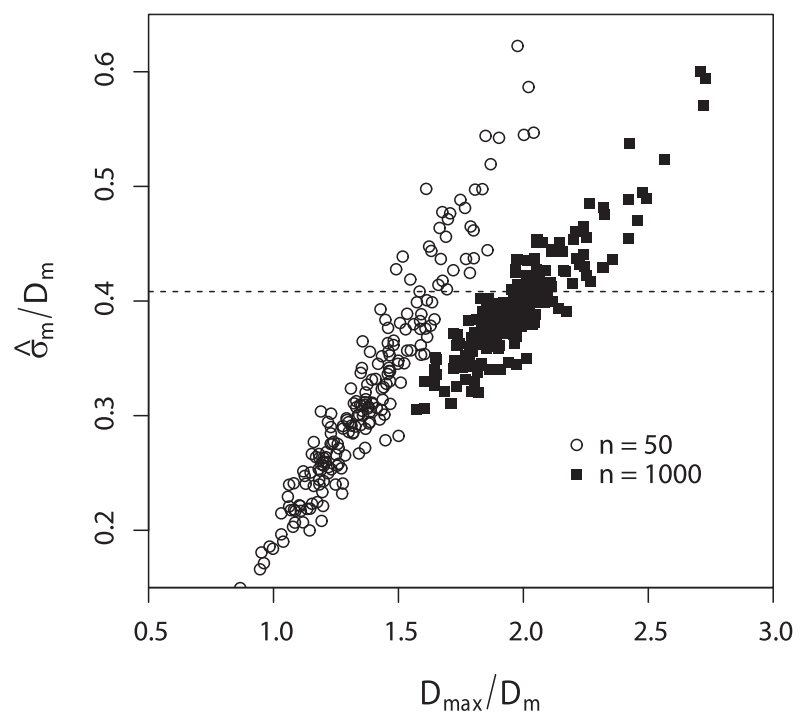

FIG. 9. Illustration of strong correlation of sample estimates of mass standard deviation $\hat{\sigma}_{m}$ with size of largest drop in the sample $D_{\max }$. The composite scatterplot is of normalized estimates for samples of 50 and 1000 drops from a gamma DSD with population shape parameter $\mu=2$; the dashed line indicates the normalized population value of $\sigma_{m}$

Underestimates of $\sigma_{m}$ lead to overestimates of $\mu$, and Fig. 12 illustrates the slow moderation of the skewness and bias in $\hat{\mu}_{345}$ as $\mu$ increases. Even with $\mu=10$ about $75 \%$ of the samples give overestimates for $\hat{\mu}_{345}$.

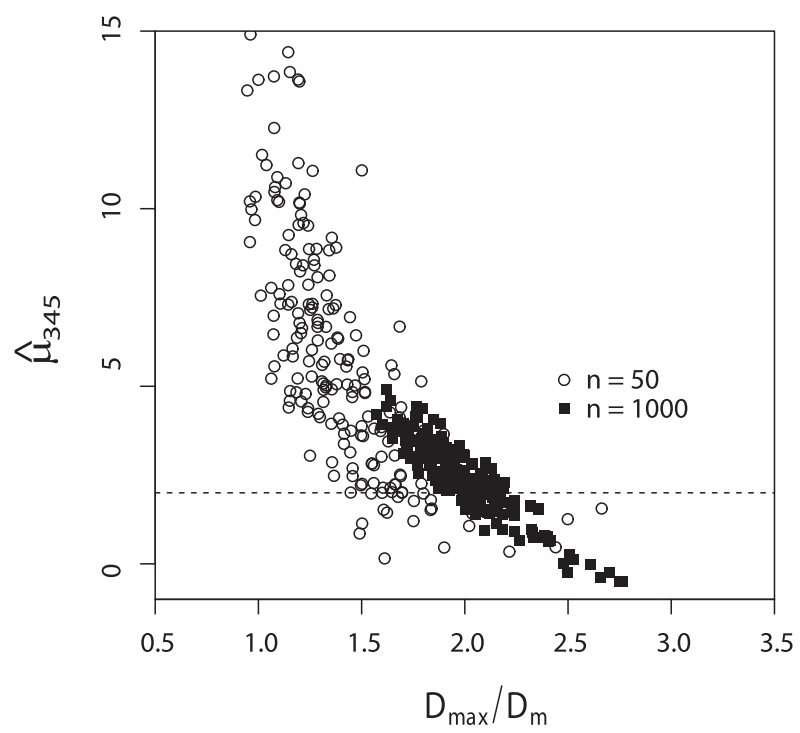

FIG. 10. Illustration of strong (nonlinear) correlation between estimates of gamma shape parameter $\mu$ from Eq. (13) and size of largest drop in the sample. The composite scatterplot is of estimates for sample sizes of 50 and 1000 drops; population shape parameter $\mu=2$. The dashed line indicates the normalized population value.

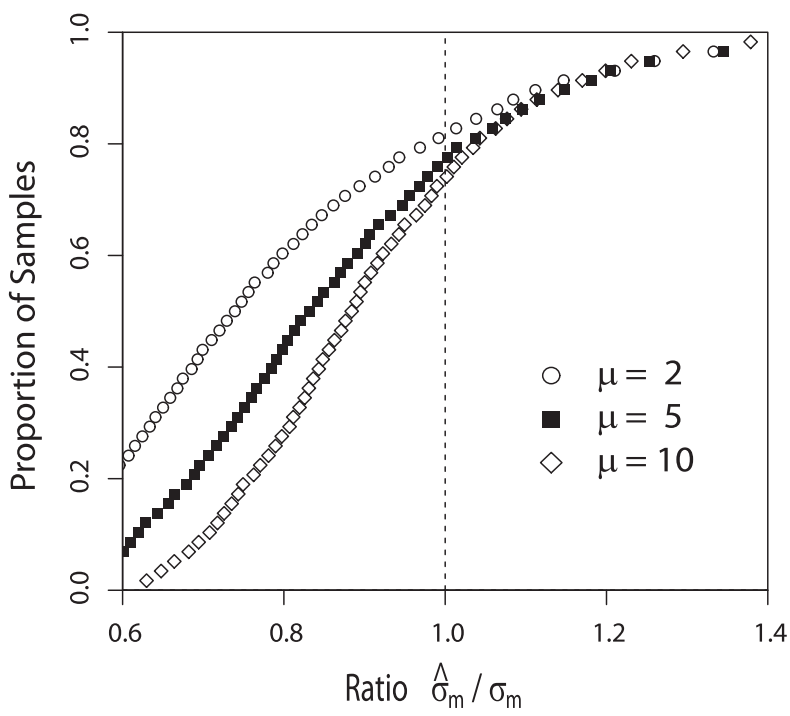

FIG. 11. Illustration of slow decrease in skewness of sample estimates of the gamma DSD parameter $\sigma_{m}$ with increasing population values of $\mu$. Cumulative frequency distributions are of normalized estimates for different population values of $\mu$; sample sizes are 50 drops.

The relative bias and RMSE in the $\hat{\mu}_{345}$ values decrease as $\mu$ increases, but the absolute bias changes little and the absolute RMSE actually increases as $\mu$ increases (this also holds true for sample sizes of 1000 drops).

Table 2 summarizes the sampling statistics for the normalized gamma DSD parameters, including those previously discussed, estimated from samples of 50 and

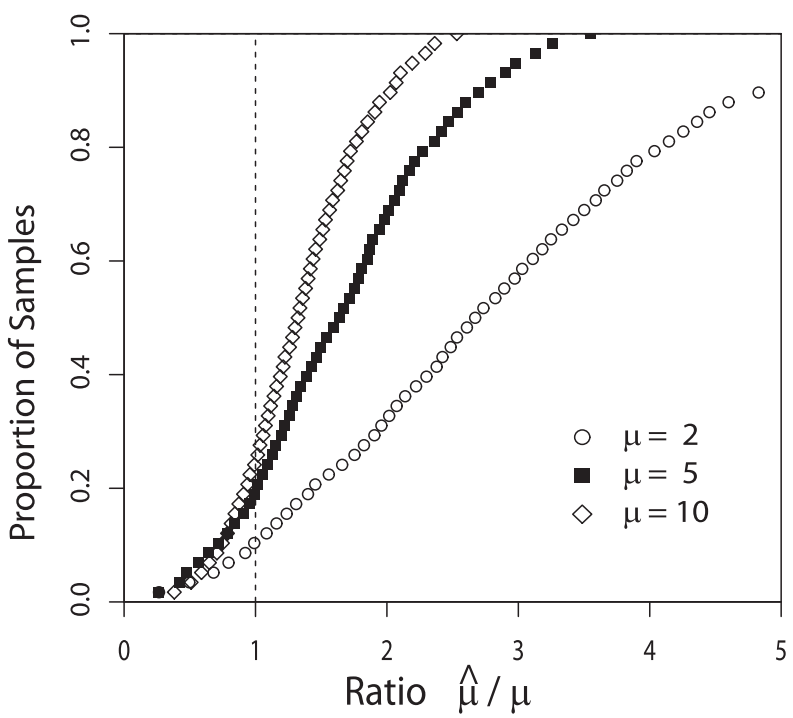

FIG. 12. Illustration of slow decrease in skewness of sample estimates of the gamma DSD shape parameter $\mu$ according to Eq. (13) with increasing population values of $\mu$. Cumulative frequency distributions are of normalized estimates for different population values of $\mu$; sample sizes are 50 drops. 
TABLE 2. Summary statistics for the normalized Eq. (13) (m345) estimates of the indicated gamma DSD parameters for different population values of $\mu$.

\begin{tabular}{|c|c|c|c|c|c|c|c|c|c|c|}
\hline \multirow[b]{2}{*}{ Sample size } & \multirow[b]{2}{*}{ Estimate } & \multicolumn{3}{|c|}{$\mu=2$} & \multicolumn{3}{|c|}{$\mu=5$} & \multicolumn{3}{|c|}{$\mu=10$} \\
\hline & & Median & Mean & RMSE & Median & Mean & RMSE & Median & Mean & RMSE \\
\hline \multirow[t]{4}{*}{50} & $\hat{\mu} / \mu$ & 2.78 & 3.01 & 2.58 & 1.58 & 1.70 & 1.05 & 1.32 & 1.35 & 0.62 \\
\hline & $\hat{\boldsymbol{\sigma}}_{m} / \sigma_{m}$ & 0.73 & 0.78 & 0.33 & 0.83 & 0.87 & 0.26 & 0.89 & 0.92 & 0.22 \\
\hline & $\hat{N}_{w} / N_{w}$ & 1.30 & 1.33 & 0.61 & 1.12 & 1.12 & 0.30 & 1.05 & 1.05 & 0.17 \\
\hline & $\hat{D}_{m} / D_{m}$ & 0.926 & 0.953 & 0.169 & 0.968 & 0.979 & 0.105 & 0.985 & 0.990 & 0.068 \\
\hline \multirow[t]{4}{*}{1000} & $\hat{\mu} / \mu$ & 1.29 & 1.25 & 0.61 & 1.08 & 1.06 & 0.26 & 1.04 & 1.03 & 0.14 \\
\hline & $\hat{\boldsymbol{\sigma}}_{m} / \sigma_{m}$ & 0.95 & 0.97 & 0.14 & 0.98 & 0.99 & 0.09 & 0.98 & 0.99 & 0.06 \\
\hline & $\hat{N}_{w} / N_{w}$ & 1.03 & 1.03 & 0.15 & 1.01 & 1.01 & 0.08 & 1.00 & 1.01 & 0.04 \\
\hline & $\hat{D}_{m} / D_{m}$ & 0.993 & 0.997 & 0.050 & 0.997 & 0.999 & 0.027 & 0.998 & 0.999 & 0.016 \\
\hline
\end{tabular}

1000 drops from populations with the indicated shape parameters. All the biases and RMSEs decrease as the sample size increases or as the population $\mu$ increases. The biases in estimates of $\sigma_{m}$ are $3 \%$ or less with sample sizes of 1000 drops but are greater with smaller sample sizes. The biases in $\hat{\mu}$ are more substantial throughout the table, and the RMSEs are greater than $25 \%$ except for the case with population $\mu=10$ and sample sizes of 1000 drops. For all of the combinations tabulated, the biases in $\hat{D}_{m}$ are less than $5 \%$, and they are less than $1 \%$ with sample sizes of 1000 drops. Those in $\hat{N}_{w}$ are somewhat greater but are $5 \%$ or less with sample sizes of 1000 drops or with $\mu=10$ and sample sizes of greater than 50 drops. The correlation between the sample estimates of $\sigma_{m}$ and $D_{m}$ remains strong ( 0.80 or greater) as the population $\mu$ increases (e.g., Fig. 13).

\section{Discussion and conclusions}

The intent of introducing $\sigma_{m}$ as a DSD parameter was to circumvent the problems associated with sampling small raindrops with the available disdrometers when trying to estimate the parameters. With parameters that depend on the third and higher moments of the disdrometer-estimated DSD, that purpose has been accomplished. However, as the simulations in section 3 illustrate the result has been to trade the difficulties with sampling small drops for other, and perhaps more serious, difficulties in trying to adequately sample the large drops. Very large samples are required to represent the large-drop tail of a DSD well, and the strong relationship between the parameter estimates and the size of the largest drop in the sample (e.g., Figs. 9 and 10) suggests that even samples of 1000 drops may not be sufficient. Samples as small as 50 drops are certainly not adequate to the task.

The mathematical analysis in section 2 shows that the disdrometer DSD parameter estimators in the procedure outlined in Williams et al. (2014) are essentially moment estimators, presented in a different format. As such, the estimates can be subject to strong biases and significant errors. Earlier work (e.g., Smith et al. 2009) has shown that the biases and errors increase as higher-order moments are used. Thus, for example, the procedure that uses the third, fourth, and fifth moments of the sample DSDs would be inferior to the method using the second, third, and fourth moments treated in Smith et al. (although that method cannot include the parameter $\sigma_{m}$ ).

With respect to the specific case of gamma DSDs, the simulation results in section 3 clearly show that there is strong bias and substantial error in estimates of the shape parameter $\mu$ obtained from the estimates of $\sigma_{m}$. This is consistent with earlier results on the use of moment estimators for gamma DSD parameters (e.g., Smith et al. 2009). Figures 5 and 7 and Table 1

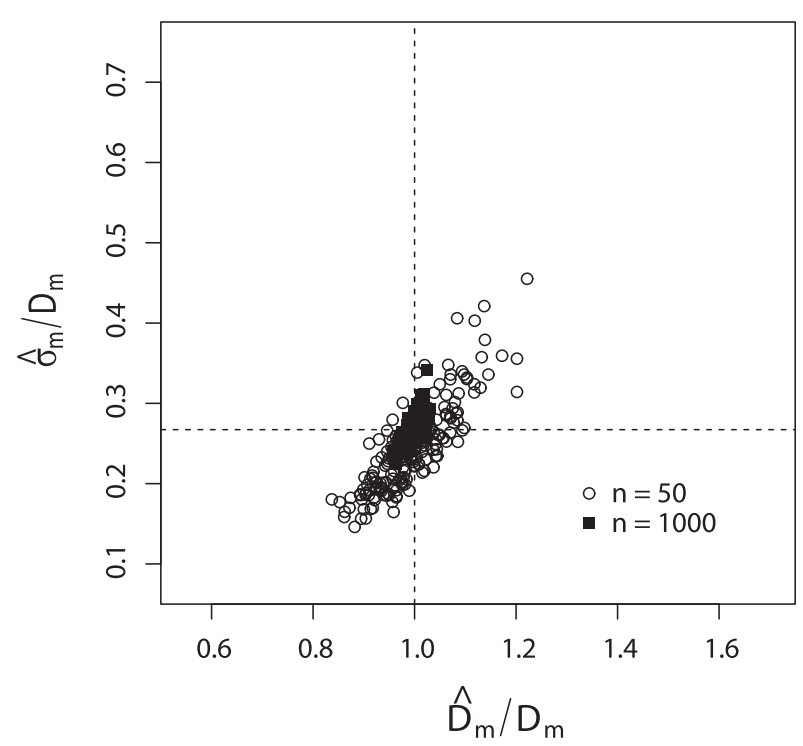

FIG. 13. Illustration of the statistical correlation between sample estimates of gamma DSD parameters $\hat{\sigma}_{m}$ and $\hat{D}_{m}$ for samples from a narrower gamma DSD. Population shape parameter $\mu=10$; sample sizes are 50 and 1000 drops. Dashed lines indicate normalized population values. 
demonstrate the superiority of maximum likelihood estimators when the form of the DSD function is specified. Use of $\sigma_{m}$ as a parameter would not be a good approach for most applications that involve estimating DSD parameters from disdrometer data when the gamma DSD model is employed, nor would it be a good choice when some other mathematical model of the DSD amenable to maximum likelihood analysis is specified.

Acknowledgments. This work was supported by the South Dakota School of Mines and Technology.

\section{REFERENCES}

Alonge, A., and T. Afullo, 2012: Seasonal analysis and prediction of rainfall effects in eastern South Africa at microwave frequencies. Prog. Electromagn. Res., 40, 279-303, https:// doi.org/10.2528/PIERB12020305.

Best, A. C., 1950: The size distribution of raindrops. Quart. J. Roy. Meteor. Soc., 76, 16-36, https://doi.org/10.1002/qj.49707632704.

Bradley, S. G., and C. D. Stow, 1974: The measurement of charge and size of raindrops. Part II: Results and analysis at ground level. J. Appl. Meteor., 13, 131-147, https://doi.org/10.1175/ 1520-0450(1974)013<0131:TMOCAS > 2.0.CO;2.

Bringi, V. N., and V. Chandrasekar, 2001: Polarimetric Doppler Weather Radar: Principles and Applications. Cambridge University Press, $636 \mathrm{pp}$.

Caracciolo, C., F. Prodi, A. Battaglia, and F. Porcu, 2006: Analysis of the moments and parameters of a gamma DSD to infer precipitation properties. Atmos. Res., 80, 165-186, https://doi.org/ 10.1016/j.atmosres.2005.07.003.

Feingold, G., and Z. Levin, 1986: The lognormal fit to raindrop spectra from frontal convective clouds in Israel. J. Climate Appl. Meteor., 25, 1346-1363, https://doi.org/10.1175/1520-0450(1986) 025<1346:TLFTRS $>2.0$.CO;2.

Hou, A. Y., and Coauthors, 2014: The Global Precipitation Mission. Bull. Amer. Meteor. Soc., 95, 701-722, https://doi.org/ 10.1175/BAMS-D-13-00164.1.

Johnson, R. W., D. V. Kliche, and P. L. Smith, 2011: Comparison of estimators for parameters of gamma distributions with lefttruncated samples. J. Appl. Meteor. Climatol., 50, 296-310, https://doi.org/10.1175/2010JAMC2478.1.

$\ldots, \ldots$, and — 2014: Maximum likelihood estimation of gamma parameters for coarsely-binned and truncated raindrop size data. Quart. J. Roy. Meteor. Soc., 140, 1245-1256, https://doi.org/10.1002/qj.2209.

Kliche, D. V., 2007: Raindrop size distribution functions: An empirical approach. Ph.D. dissertation, South Dakota School of Mines and Technology, $211 \mathrm{pp}$.

Kozu, T., and K. Nakamura, 1991: Rainfall parameter estimation from dual-radar measurements combining reflectivity profile and path-integrated attenuation. J. Atmos. Oceanic Technol., 8, 259-270, https://doi.org/10.1175/1520-0426(1991)008<0259: RPEFDR $>2.0 . \mathrm{CO} ; 2$.

Mallet, C., and L. Barthes, 2009: Estimation of gamma raindrop size distribution parameters: Statistical fluctuations and estimation errors. J. Atmos. Oceanic Technol., 26,1572-1584, https://doi.org/ 10.1175/2009JTECHA1199.1.

Markowitz, A. H., 1976: Raindrop size distribution expressions. J. Appl. Meteor., 15, 1029-1031, https://doi.org/10.1175/ 1520-0450(1976)015<1029:RSDE > 2.0.CO;2.
Marshall, J. S., and W. M. Palmer, 1948: The distribution of raindrops with size. J. Meteor., 5, 165-166, https://doi.org/10.1175/ 1520-0469(1948)005<0165:TDORWS >2.0.CO;2.

Rice, J., 1995: Mathematical Statistics and Data Analysis, 2nd ed. Wadsworth, 602 pp.

Robertson, C. A., and J. G. Fryer, 1970: The bias and accuracy of moment estimators. Biometrika, 57, 57-65, https://doi.org/ 10.1093/biomet/57.1.57.

Sekhon, R. S., and R. C. Srivastava, 1970: Snow size spectra and radar reflectivity. J. Atmos. Sci., 27, 299-307, https://doi.org/ 10.1175/1520-0469(1970)027<0299:SSSARR > 2.0.CO;2.

Smith, P. L., 2016: Sampling issues in estimating radar variables from disdrometer data. J. Atmos. Oceanic Technol., 33, 23052313, https://doi.org/10.1175/JTECH-D-16-0040.1.

_- D. V. Kliche, and R. W. Johnson, 2009: The bias and error in moment estimators for parameters of drop size distribution functions: Sampling from gamma distributions. J. Appl. Meteor. Climatol., 48, 2118-2126, https://doi.org/10.1175/ 2009JAMC2114.1.

Thurai, M., P. Gatlin, V. N. Bringi, W. Petersen, P. Kennedy, B. Notaros, and L. Carey, 2017: Toward completing the raindrop size spectrum: Case studies involving 2D-video disdrometer, droplet spectrometer, and polarimetric radar measurements. J. Appl. Meteor. Climatol., 56, 877-896, https://doi.org/10.1175/ JAMC-D-16-0304.1.

Tokay, A., and D. A. Short, 1996: Evidence from tropical raindrop spectra of the origin of rain from stratiform versus convective clouds. J. Appl. Meteor., 35, 355-371, https://doi.org/10.1175/ 1520-0450(1996)035<0355:EFTRSO > 2.0.CO;2.

Ulbrich, C. W., 1983: Natural variation in the analytical form of the raindrop size distribution. J. Climate Appl. Meteor., 22, 1764-1775, https://doi.org/10.1175/1520-0450(1983)022<1764: NVITAF $>2.0 . \mathrm{CO} ; 2$.

_ erties: Analysis methods for drop size spectra. J. Appl. Meteor., 37, 912-923, https://doi.org/10.1175/1520-0450(1998) 037<0912:RMARPA $>2.0 . \mathrm{CO} ; 2$.

Waldvogel, A., 1974: The $N_{0}$ jump of raindrop spectra. J. Atmos. Sci., 31, 1067-1078, https://doi.org/10.1175/1520-0469(1974) 031<1067:TJORS > 2.0.CO;2.

Wallis, J. R., N. C. Matalas, and J. R. Slack, 1974: Just a moment! Water Resour. Res., 10, 211-219, https://doi.org/10.1029/ WR010i002p00211.

Weber, S., 1976: The $D_{m}$ analysis of precipitation particle size distributions. M.S. thesis, Dept. of Atmospheric Sciences, South Dakota School of Mines and Technology, $80 \mathrm{pp}$.

Wilks, D. S., 1989: Rainfall intensity, the Weibull distribution, and estimation of daily surface runoff. J. Appl. Meteor., 28, 52-58, https://doi.org/10.1175/1520-0450(1989)028<0052: RITWDA $>2.0 . \mathrm{CO} ; 2$.

Williams, C. R., and Coauthors, 2014: Describing the shape of raindrop size distributions using uncorrelated raindrop mass spectrum parameters. J. Appl. Meteor. Climatol., 53, 12821296, https://doi.org/10.1175/JAMC-D-13-076.1.

_ - and Coauthors, 2015: Reply to "Comments on 'Describing the shape of raindrop size distributions using uncorrelated raindrop mass spectrum parameters."' J. Appl. Meteor. Climatol., 54, 1977-1982, https://doi.org/10.1175/JAMC-D-150058.1.

Ye, Z.-S., and N. Chen, 2017: Closed-form estimators for the gamma distribution derived from likelihood equations. Amer. Stat., 71, 177-181, https://doi.org/10.1080/00031305. 2016.1209129. 